\title{
STONE-CECH REMAINDERS WHICH MAKE CONTINUOUS IMAGES NORMAL
}

\author{
WILLIAM FLEISSNER AND RONNIE LEVY
}

(Communicated by Dennis K. Burke)

\begin{abstract}
If $f$ is a continuous surjection from a normal space $X$ onto a regular space $Y$, then there are a space $Z$ and a perfect map $b f: Z \rightarrow Y$ extending $f$ such that $X \subset Z \subset \beta X$. If $f$ is a continuous surjection from normal $X$ onto Tychonov $Y$ and $\beta X \backslash X$ is sequential, then $Y$ is normal. More generally, if $f$ is a continuous surjection from normal $X$ onto regular $Y$ and $\beta X \backslash X$ has the property that countably compact subsets are closed (this property is called $C$-closed), then $Y$ is normal. There is an example of a normal space $X$ such that $\beta X \backslash X$ is $C$-closed but not sequential. If $X$ is normal and $\beta X \backslash X$ is first countable, then $\beta X \backslash X$ is locally compact.
\end{abstract}

We began the study of the class of spaces ACRIN (all continuous regular images normal) in [FL], where we showed that if $X$ is a normal space such that $\beta X \backslash X$ is finite, then $X$ is ACRIN. Here, we give more general conditions on $\beta X \backslash X$ which imply that a normal space is ACRIN.

For a Tychonov space $X$, we denote the Stone-Cech compactification of $X$ by $\beta X$. We call a space $Z$ such that $X \subset Z \subset \beta X$ an intermediate space. If $X$ and $Y$ are Tychonov spaces and $f: X \rightarrow Y$ is a continuous map, we denote by $\beta f: \beta X \rightarrow \beta Y$ the Stone extension of $f$.

Let us recall some well-known properties of maps.

1. Lemma. Let $f: X \rightarrow Y$ be a continuous surjection. (a) If $X$ is normal and $f$ is a closed mapping, then $Y$ is normal.

(b) If $X$ is countably compact, then $Y$ is countably compact.

(c) If $f$ is perfect and $Y$ is countably compact, then $X$ is countably compact.

(d) If $\beta f \rightarrow(\beta X \backslash X)=\beta Y \backslash Y$, then $f$ is perfect.

Let us fix a continuous surjection $f: X \rightarrow Y$ from a normal space to a regular space. Let $b f: P \rightarrow Y$ be a perfect map extending $f$, where $P$ is an intermediate space. If $Y$ is Tychonov, we will set $P=\beta f^{\leftarrow}(Y)$ and

Received by the editors July 18,1988 and, in revised form, October $11,1988$.

1980 Mathematics Subject Classification (1985 Revision). Primary 54C05, 54D15.

Key words and phrases. Stone-Cech remainder, continuous image, normal, ACRIN, C-closed, normality inducing.

The first author gratefully acknowledges support from NSF Grant DMS-8603227 and University of Kansas General Research allocation \#3762-XO-0038. 
$b f=\beta f \mid P$. We defer the discussion of how to define $P$ and $b f$ in the case when $Y$ is regular but not Tychonov. From Lemma 1 , we see that if $P$ is normal, then $Y$ is normal. Thus, we seek conditions which imply that $P$ is normal.

One such condition is to assert that every intermediate space $Z$ is normal. Barr and Hajek introduced this notion in $[\mathrm{BH}]$ and called it normality inducing. Further, they showed that if $X$ is normality inducing, then $X$ is countably compact, and they showed that $X$ is normality inducing if and only if every compact subset of $\beta X \backslash X$ is finite.

A condition which implies that an intermediate space $Z$ is normal is that $Z \backslash X$ is closed in $\beta X \backslash X$. Then $Z$ is normal because it is the union of the normal space $X$ and the compact space $\mathrm{Cl}_{\beta X}(Z \backslash X)$. (See [FL, Lemma 1.1(c)].) Thus, if $X$ is normal and $\beta X \backslash X$ is finite, or more generally, discrete, then $X$ is normality inducing, and hence ACRIN.

Let us assume that $X$ is countably compact. Again from Lemma 1, we see that $P$ is countably compact. Thus, it is not necessary that every intermediate space $Z$ be normal; it is enough to require that countably compact intermediate spaces be normal.

2. Proposition. If $\beta X \backslash X$ is sequential and $Y$ is Tychonov, then $P \backslash X$ is closed in $\beta X \backslash X$. Hence, $Y$ is normal.

Proof. Suppose that $q \in \beta X \backslash X$ and there is a sequence $\left(p_{n}\right)_{n \in \omega}$ in $P \backslash X$ converging to $q$. By the definition of $P$, each $\beta f\left(p_{n}\right)$ is an element of $Y$. Since $Y$ is countably compact, $\left(\beta f\left(p_{n}\right)\right)_{n \in \omega}$ has a cluster point $y$ in $Y$. By continuity, $\left(\beta f\left(p_{n}\right)\right)_{n \in \omega}$ converges to $y$ and $y=\beta f(q)$. Therefore, $q \in P$, and $P \backslash X$ is sequentially closed in $\beta X \backslash X$. Because $\beta X \backslash X$ is sequential, $P \backslash X$ is closed in $\beta X \backslash X$.

A space is called $C$-closed if every countably compact subset is closed. (See [IN].) For example, sequential spaces are $C$-closed. Countable spaces, which need not be sequential, and $P$-spaces, which are sequential if and only if they are discrete, are $C$-closed. If $X$ is normal and not countably compact, then $X$ contains a closed copy of $\omega$. Thus, $\beta X \backslash X$ contains a closed copy of $\beta \omega \backslash \omega$. Therefore, $\beta X \backslash X$ is not $C$-closed-it contains the nonclosed countably compact subset $(\beta \omega \backslash \omega) \backslash\{p\}$ where $p \in \beta \omega \backslash \omega$.

3. Theorem. If $\beta X \backslash X$ is $C$-closed, then every countably compact intermediate space $Z$ is normal. Hence $X$ is ACRIN.

Proof. Towards a contradiction, assume that $H$ and $K$ are disjoint closed subsets of a countably compact intermediate space $Z$ and that $q \in\left[\mathrm{Cl}_{\beta X}(H) \cap\right.$ $\left.\mathrm{Cl}_{\beta X}(K)\right] \backslash Z$. If $q$ were an element of $\mathrm{Cl}_{\beta X}(H \cap X) \cap \mathrm{Cl}_{\beta X}(K \cap X)$, then $X$ would not be normal. Assume without loss of generality that $q \notin \mathrm{Cl}_{\beta X}(H \cap X)$. By regularity, there is an open subset $U$ of $\beta X$ with $\mathrm{Cl}_{\beta X}(H \cap X) \subseteq U \subseteq$ $\mathrm{Cl}_{\beta X}(U) \subseteq \beta X \backslash\{q\}$. Then $H \backslash U$ is a countably compact subset of $\beta X \backslash X$ 
(since it is a closed subset of $Z$ ), and since $\beta X \backslash X$ is assumed to be $C$-closed, $q \in \mathrm{Cl}_{\beta X \backslash X}(H \backslash X)=H \backslash U \subseteq Z$, contradicting $q \notin Z$.

If $Y$ is a locally compact space and $\alpha Y=Y \cup\{\infty\}$ is its one-point compactification, then for large enough cardinal $\gamma$, the subspace $(\alpha Y \times \gamma) \cup(\infty, \gamma)$ of $\alpha Y \times(\gamma+1)$ is a normal space whose Stone-Cech remainder is $Y$. Thus, every locally compact space is a remainder of a normal space. The following result limits the applicability of Theorem 3 to exactly the locally compact spaces if $\beta X \backslash X$ is first countable.

4. Proposition. If $X$ is normal and $\beta X \backslash X$ is first countable, then $\beta X \backslash X$ is locally compact.

Proof. Towards a contradiction, suppose that $p \in \beta X \backslash X$ has a countable nested base in $\beta X \backslash X$ such that for all $n, C l_{\beta X \backslash X}\left(B_{n}\right)$ is not compact. Since $\mathrm{Cl}_{\beta X}\left(B_{n}\right)$ is compact, we may choose distinct $x_{n} \in \mathrm{Cl}_{\beta X}\left(B_{n}\right) \cap X$. Then $\left\{x_{2 n}: n \in \omega\right\}$ and $\left\{x_{2 n+1}: n \in \omega\right\}$ are disjoint closed subsets of $X$, both of whose closures in $\beta X$ contain $p$. This contradicts the normality of $X$.

There are normal spaces whose Stone-Cech compactifications are $C$-closed, but not locally compact. For example, it is not hard to show that if $E$ is an $\eta_{1}$ set with the order topology and $X$ is the set of non- $P$-points of the Dedekind compactification of $E$, then $\beta X \backslash X$ is a $P$-space without isolated points, and hence is $C$-closed but not locally compact. Since $X$, a linearly ordered space is normal, it follows from Theorem 3 that every continuous image of $X$ is normal.

We now give alternative definitions of $b f$ and $P$ which require only that $Y$ be regular. The general situation is this: $f$ is a continuous surjection from the normal space $X$ to a regular space $Y$, and $Z$ is a Hausdorff extension of $X$, that is, a Hausdorff space which contains $X$ as a dense subspace. For $p \in Z$, define $\mathscr{N}_{p}=\{N \cap X: N$ is a neighborhood of $p$ in $Z\}$. Let $G$ be $\mathrm{Cl}_{Z \times Y}($ graph $f)$.

5. Lemma. Let $f, X, Y, Z$, and $G$ be as above. (a) For $p \in Z \backslash X, f \cup$ $\{(p, y)\}$ is continuous if and only if $f\left[\mathcal{N}_{p}\right]$ converges to $y$.

(b) If for all $p \in Z, f \cup\left\{\left(p, y_{p}\right)\right\}$ is a continuous function, then $f \cup\left\{\left(p, y_{p}\right)\right.$ : $p \in Z\}$ is a continuous function.

(c) $(p, y) \in G$ if and only if $f\left[\mathcal{N}_{p}\right]$ adheres to $y$.

Further assume that $X$ is normal and $Z$ is $\beta X$.

(d) If $f\left[\mathcal{N}_{p}\right]$ adheres to $y$, then $f\left[\mathcal{N}_{p}\right]$ converges to $y$.

(e) Hence, $G$ is the graph of a function $b f$ and $b f$ is perfect.

Proof. (a) and (b) are [PW, 4.1(1) and 4.1(n)]; (c) is routine. Proof of (d): Because $X$ is normal and $Z=\beta X$, we may consider points of $Z$ to be ultrafilters of closed subsets of $X$ and basic open sets have the form $N(F)=$ $\{q \in \beta X: F \notin q\}$. We prove the contrapositive. Suppose that $f\left[\mathcal{N}_{p}\right]$ does not converge to $y$. There is a neighborhood $V$ of $y$ which does not contain $f(N)$ 
for any $N \in \mathscr{N}_{p}$. Because $Y$ is regular, there is an open $W$ such that $y \in W$ and $\mathrm{Cl}_{Y} W \subseteq V$. Consider $H=f^{\leftarrow}\left(\mathrm{Cl}_{Y} W\right)=\left\{x \in X: f(x) \in \mathrm{Cl}_{Y} W\right\}$. Then $N(H) \in \mathscr{N}_{p}$ and $f(N(H)) \cap W=\phi$. Thus, $f\left[\mathscr{N}_{p}\right]$ does not adhere to $y$.

We have arranged things so that the proof of (e) is easy. By (c), (d), and the fact that points of convergence are unique in Hausdorff spaces, $b f$ is a function. Continuity follows from the previous parts. Because $Z$ is compact, the projection onto $Y$ is a closed map; $b f$ is the restriction of projection to the closed set $G$, so it is also closed. Finally, $b f^{\leftarrow}\{y\}$ is $G \cap(Z \times\{y\})$, the intersection of a closed set and a compact set.

We wish to thank Jack Porter for discussions which transformed our original ad hoc, dot and circle construction into Lemma 5.

We close with some questions.

Question 1. If $X$ is normal and $\beta X \backslash X$ is countable, is $\beta X \backslash X$ sequential? If $X$ is normal and $\beta X \backslash X$ is sequential, is $\beta X \backslash X$ locally compact?

Question 2. If $X$ is normal and $\beta X \backslash X$ has countable tightness, is $X$ ACRIN? Is there, without extra axioms of set theory, a regular space of countable tightness which is not $C$-closed? (Balogh proved, assuming PFA, that locally compact spaces of countable tightness are $C$-closed. Fedorchuk constructed, assuming $\diamond$, a Tychonov space of countable tightness which is not $C$-closed.)

\section{REFERENCES}

[BH] D. Barr and D. Hajek, Normality inducing spaces, Gen. Top. Appl. 7 (1977), 89-97.

[FL] W. Fleissner and R. Levy, Ordered spaces all of whose continuous images are normal, Proc. Amer. Math. Soc. 355 (1989), 231-235.

[IN] M. Ismail and P. Nyikos, On spaces in which countably compact sets are closed and hereditary properties, Topology Appl. 11 (1980), 281-290.

[PW] J. Porter and G. Woods, Extensions and absolutes of Hausdorff spaces, Springer-Verlag, New York, 1988.

Department of Mathematics, University of Kansas, Lawrence, Kansas 66045

Department of Mathematics, George Mason University, FairfaX, Virginia 22030 